\title{
CALIBRAÇÃO DE MODELO HIDRÁULICO DE REDE DE ABASTECIMENTO DE ÁGUA VIA MODELO INVERSO
}

\author{
Hydraulic Model Calibration of Water Supply System Via Inverse Model
}

\author{
Agne Pereira da Cunha ${ }^{1}$, Cryslara de Souza Lemes ${ }^{2}$, Willian Teles Pinto ${ }^{3}$, Alexandre Kepler Soares ${ }^{4}$ \\ Recebido em 14 de maio de 2015; recebido para revisão em 03 de junho de 2015; aceito em 09 de julho de 2015; \\ disponivel on-line em 25 de julho de 2015.
}

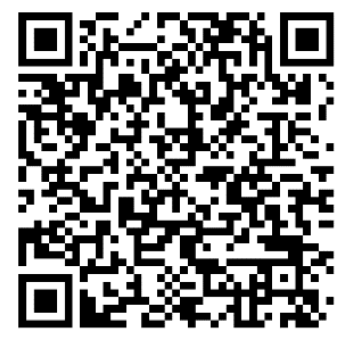

PALAVRAS CHAVE:

Sistema de distribuição de água;

Modelagem hidráulica;

Algoritmos genéticos;

Perdas de água.

\section{KEYWORDS:}

Water distribution system;

Hydraulic modelling;

Genetic algorithms;

Water losses.

* Contato com os autores:

1 e-mail : agne__@hotmail.com ( A. P. Cunha )

Graduanda em Engenharia Ambiental e Sanitária, Universidade Federal de Goiás.

2e-mail : cryslara_sl@hotmail.com (C. S. Lemes )

Engenheira Ambiental e Sanitarista pela Universidade Federal de Goiás.

${ }^{3}$ e-mail : willianteles@hotmail.com ( W. T. Pinto )

Graduando em Engenharia Ambiental e Sanitária, Universidade Federal de Goiás.

${ }^{4}$ e-mail : aksoares@gmail.com ( A. K. Soares)

Engenheiro Civil Doutor, Professor Adjunto da Universidade Federal de Goiás. 


\section{INTRODUÇÃO}

Os recursos naturais vêm se tornando cada vez mais escassos e os custos para o tratamento da água, de modo a deixá-la adequada para consumo humano, estão cada vez maiores. Devido a este fato, um bom gerenciamento dos recursos hídricos se torna indispensável para a preservação deste bem.

Tsutiya (2006) afirma que, durante o funcionamento dos SAAs (Sistemas de Abastecimento de Água), desde a captação no manancial até a entrega da água tratada ao consumidor, ocorrem várias perdas de água que em grande parte são causadas pela ineficiência na operação e manutenção desses sistemas, além da inadequada gestão comercial das companhias de saneamento.

De acordo com Garcia et al. (2013), as perdas podem ser definidas como a diferença entre a quantidade de água produzida e a quantidade efetivamente entregue aos consumidores, e podem ser classificadas em perdas reais e aparentes (físicas e não físicas). As perdas reais estão relacionadas aos vazamentos nas tubulações e conexões da rede de abastecimento e as aparentes originam-se de ligações não cadastradas ou clandestinas e erros de medição.

Para suprir a demanda de água para abastecimento da população, é necessário reduzir o índice de perdas fazendo um gerenciamento eficaz dos SAAs. Viegas et al. (2002) afirmam que um dos instrumentos disponíveis para este gerenciamento é a modelagem hidráulica das redes de distribuição de água, que proporciona o aumento da eficiência, seja na fase de projeto do sistema de abastecimento ou no seu controle operacional. Segundo Evangelista (2004), especialistas do mundo todo têm se dedicado ao desenvolvimento de modelos matemáticos computacionais confiáveis para permitir simulações do comportamento desses sistemas.

Atualmente, podem ser encontrados vários softwares utilizados para a modelagem de SAAs, que possibilitam a redução de custos e aumentam a sua confiabilidade. Um desses softwares é o EPANET, desenvolvido pela United
States Environmental Protection Agency (EPA). Viegas et al. (2002) explicam que o modelo EPANET 2.0 (Rossman, 2000) pode ser usado para diferentes tipos de aplicações em análise de sistemas de distribuição de água, inclusive para a simulação e calibração de modelos hidráulicos, avaliando os parâmetros de demanda e perdas de água.

Para a quantificação das perdas reais, pode ser feita a inclusão de modelos pressão versus vazamento nas análises hidráulicas. Contudo, e para que esses modelos gerem dados confiáveis, é importante que os parâmetros utilizados na avaliação sejam conhecidos, além de se fazer necessária a calibração do mesmo antes da análise do sistema. Segundo Soares et al. (2004), a calibração visa o ajuste dos parâmetros do sistema de tal forma que os desvios entre os dados simulados e observados em campo sejam minimizados.

Os procedimentos de calibração podem ser divididos em três métodos, sendo eles: método inverso, direto ou iterativo. Santos (2010) explica que o método implícito ou inverso consiste da minimização da diferença entre valores simulados e os valores correspondentes observados, por meio de uma ferramenta de otimização. De acordo com Lacerda e Carvalho (1999), a otimização é a busca da melhor solução para um dado problema e consiste em tentar várias soluções e utilizar a informação obtida neste processo de forma a encontrar soluções cada vez melhores. Entre os métodos utilizados para a otimização dos processos de calibração destacam-se os algoritmos genéticos (AGs).

Os AGs foram criados a partir dos princípios propostos por Charles Darwin sobre a evolução das espécies. "São algoritmos iterativos, em que a cada iteração a população é modificada, usando as melhores características dos elementos da geração anterior e submetendo-as aos três tipos básicos de operadores, para produzir melhores resultados" (Goldberg, 1989). De acordo com Soares e Reis (2004), apesar das inúmeras vantagens, os AGs são lentos, pois apesar de serem eficazes na avaliação do espaço de soluções, oscilam em torno da solução ótima. Assim, a fim de melhorar o tempo computacional e a eficiência do 
modelo de otimização, eles propõem a utilização de um método híbrido, onde o espaço de busca é explorado por um número de gerações usando os AGs, e para a parte final do processo, de modo a poupar tempo computacional, é utilizado o método Simplex (Nelder e Mead, 1965).

Segundo Silva et al. (2003), os dois principais tipos de AGs são os geracionais e os Steady-State. Silva e Reis (2002) ressaltam que a vantagem do uso dos AGs Steady-State é um menor tempo de processamento computacional quando comparados com os AGs Geracionais. Porém, apresentam a desvantagem de serem menos robustos.

Sendo assim, a proposta deste trabalho foi calibrar um modelo hidráulico para Zona Alta da rede de abastecimento de água do Setor Parque Santa Rita, Goiânia - GO. Para a calibração foi utilizado o modelo inverso (implícito), e como ferramenta de otimização os Algoritmos Genéticos. Além disso, foi utilizado o simulador hidráulico proposto por Soares (2003) juntamente com o software EPANET 2.0 como módulo auxiliar nas análises hidráulicas.

\section{METODOLOGIA}

\section{1. Área de estudo}

A área de estudo está localizada no conjunto Parque Santa Rita, na região sudoeste do município de Goiânia. A área é composta por dois setores, o Solar Santa Rita e o Residencial Village Santa Rita I/II (Figura 1). Segundo o cadastro da SANEAGO referente ao mês de maio de 2013, a quantidade de ligações de água e a quantidade de economias existentes no setor são listadas na Tabela 1.

Devido à sua topografia, o setor foi dividido em duas zonas, sendo elas, Zona Alta e Zonas Média e Baixa (Figura 2). O Conjunto Parque Santa Rita possui dois reservatórios e cada um deles é responsável pelo abastecimento de uma parte do setor (Tabela 2).

Dentre as zonas existentes, a Zona Alta foi selecionada com alvo deste estudo devido ao fato de possuir uma menor abrangência de área e menor quantidade de ramais prediais. Esta zona é abastecida pelo reservatório elevado, que conta com válvula reguladora de nível e uma tubulação de saída DN150. Embora a adoção de um setor de menor porte facilite as análises e as simulações durante a modelagem, não foi objetivo deste estudo propor uma metodologia para aumento da eficiência hidráulica do sistema, mas apenas avaliar a metodologia proposta. Além disso, diferentes desempenhos do modelo hidráulico podem ser obtidos em função do porte do setor em análise.

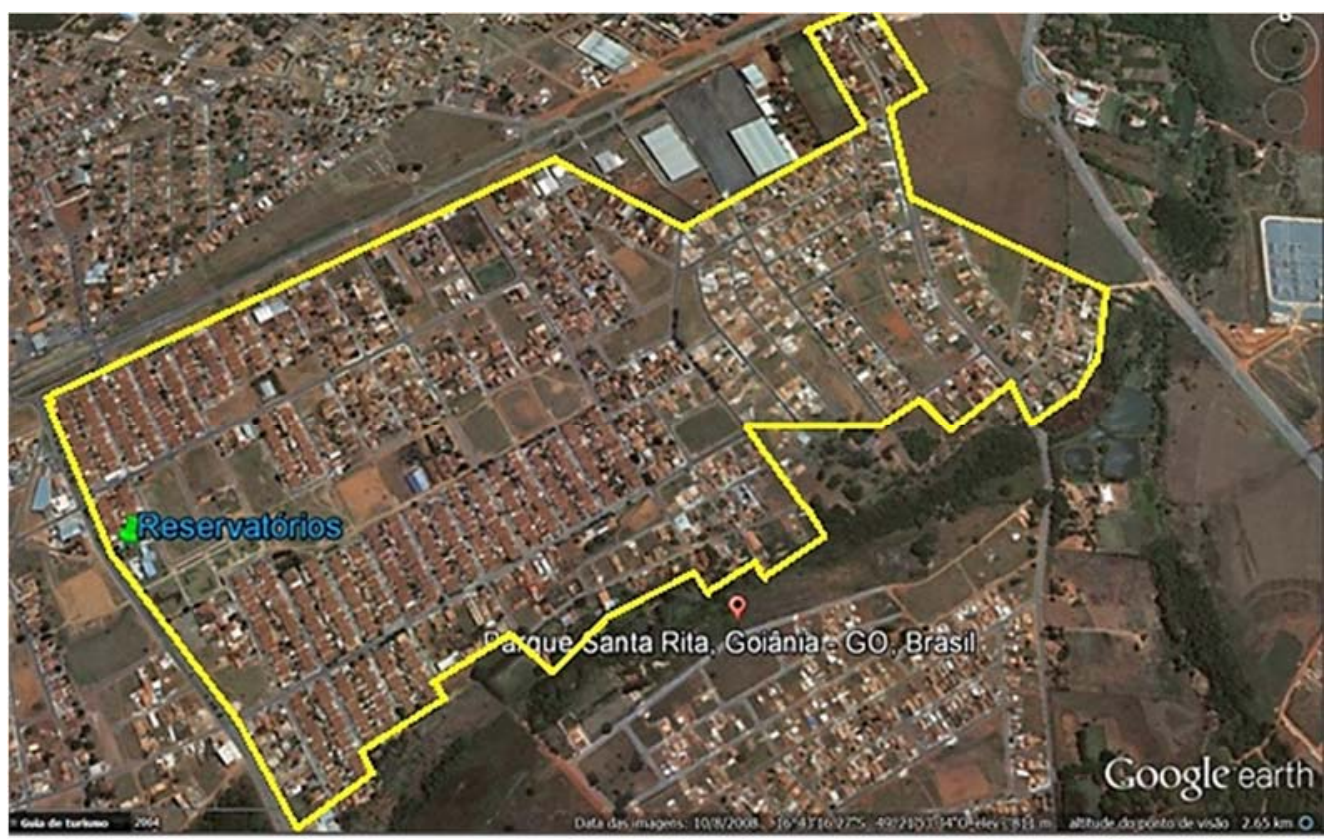

FIGURA 1: Localização do Conjunto Parque Santa Rita, Goiânia-GO. FONTE: Google Earth (2008). 
TABELA 1: Número de ligações e economias do Conjunto Parque Santa Rita, Goiânia-GO

\begin{tabular}{ccc} 
Setor & Ligações & Economias \\
\hline Res. Village Santa Rita I/II & 378 & 393 \\
\hline Solar Santa Rita & 287 & 302 \\
\hline Total & 665 & 695 \\
\hline & & FONTE: SANEAGO (2013).
\end{tabular}

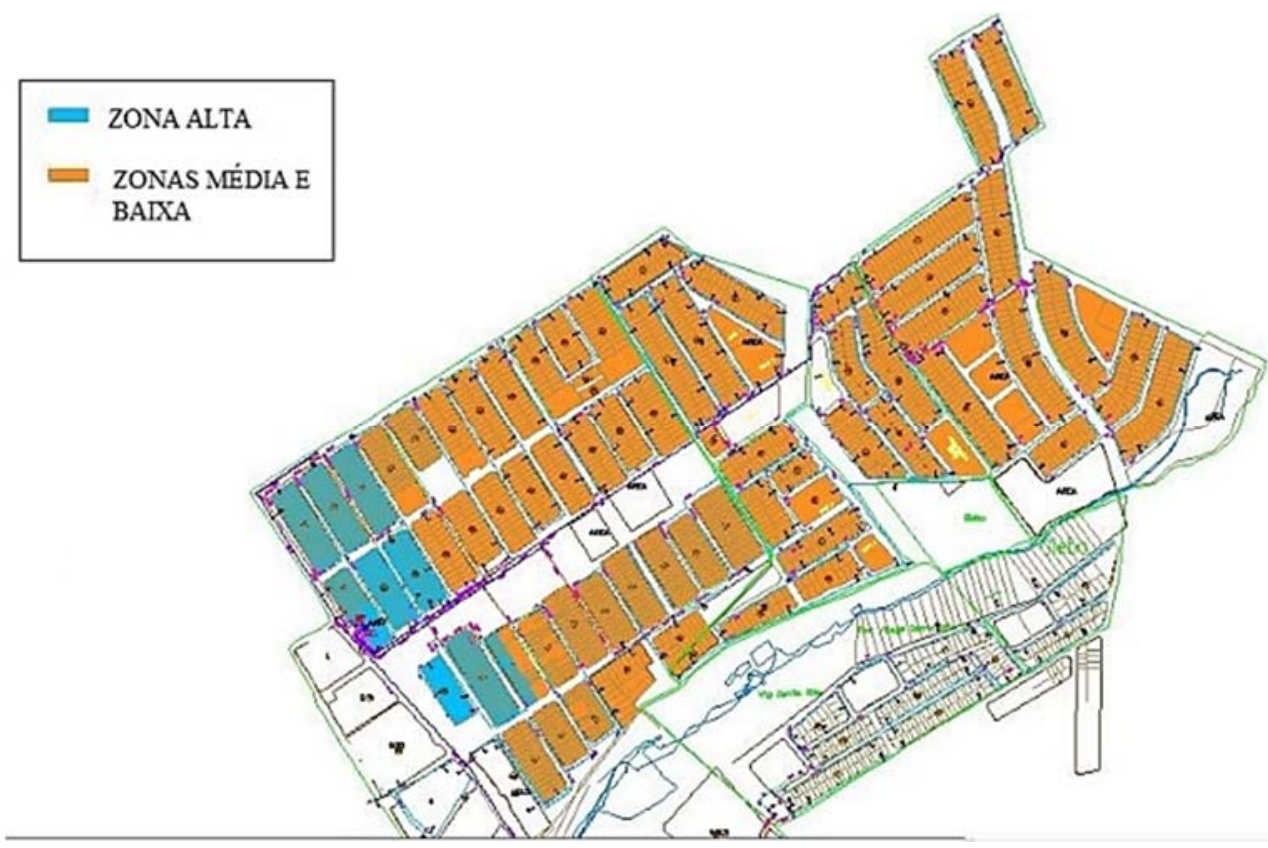

FIGURA 2: Zonas Alta, Média e Baixa do Conjunto Parque Santa Rita, Goiânia-GO FONTE: SANEAGO (2013).

TABELA 2: Reservatórios do Conjunto Parque Santa Rita, Goiânia-GO

\begin{tabular}{cccc} 
Reservatório & $\begin{array}{c}\text { Altura de } \\
\text { Reservação }(\mathbf{m})\end{array}$ & Volume $\left(\mathbf{m}^{\mathbf{3}}\right)$ & Abastecimento \\
\hline Reservatório Apoiado - RAP & 4,0 & 300 & Zonas Média e Baixa \\
\hline Reservatório Elevado - REL & 5,8 & 100 & Zona Alta \\
\hline
\end{tabular}

\subsection{Construção do modelo hidráulico}

Para a construção do modelo hidráulico da rede, foram seguidas três etapas referentes ao levantamento de dados, levantamento de equipamentos e softwares, e monitoramento de campo.

A etapa de levantamento de dados consistiu da coleta de informações e dados cadastrais da rede, como diâmetro e comprimento dos trechos, cotas topográficas dos nós e reservatórios, traçado da rede, material das tubulações, peças especiais e dimensões dos reservatórios. Todas essas informações foram disponibilizadas pela empresa SANEAGO, facilitando a realização deste trabalho.

Os equipamentos utilizados foram levantados para a execução do monitoramento de campo das vazões e pressões na rede com frequência de aquisição de dados de $1 \mathrm{~min}$. Para a coleta dos dados de vazão foi utilizado o medidor ultrassônico modelo SL1188P Portable Ultrasonic Flowmeter do fabricante "SiteLab", e, para aquisição dos dados de pressão nos nós, foram utilizados três medidores tipo datalogger sem display DMP-01-P do fabricante "Lamon Produtos 
LTDA". Para o descarregamento dos dados, foi utilizado um computador portátil e os programas que acompanharam cada aparelho. Já para as simulações e calibração, foi utilizado o software EPANET 2.0 (Rossman, 2000) para a aplicação da rotina computacional desenvolvida por SOARES (2003).

O monitoramento de campo foi realizado durante aproximadamente seis semanas. Os dados de vazão foram coletados na saída do reservatório elevado, e os de pressão coletados em três residências localizadas dentro da zona de estudo devido ao número de medidores de pressão disponíveis (três) - mais residências poderiam ser monitoradas; entretanto, a simultaneidade de aquisição dos dados não poderia ser garantida. Os pontos onde foram instalados os medidores de pressão (Figura 3) foram selecionados após observação das casas mais adequadas, onde não ocorresse nenhuma ou menor interferência externa possível. Os aparelhos foram instalados, mediante autorização dos moradores das residências, nas torneiras que tinham ligação direta com a rede de abastecimento de água, localizadas próximas aos cavaletes residenciais (Figura 4).

O medidor de vazão ultrassônico foi instalado na tubulação localizada em um poço de visita na saída do reservatório. Todos os aparelhos foram calibrados e testados antes de serem instalados em campo e, além disso, durante a instrumentação foram seguidas todas as recomendações descritas nos manuais de instalação dos equipamentos.

Assim, e de posse dos dados cadastrais da rede, foi construído no EPANET o modelo hidráulico estático, e adicionando os dados monitorados em campo, obteve-se o modelo hidráulico dinâmico da rede, contendo o padrão de consumo temporal determinado via monitoramento de vazão na entrada do sistema.

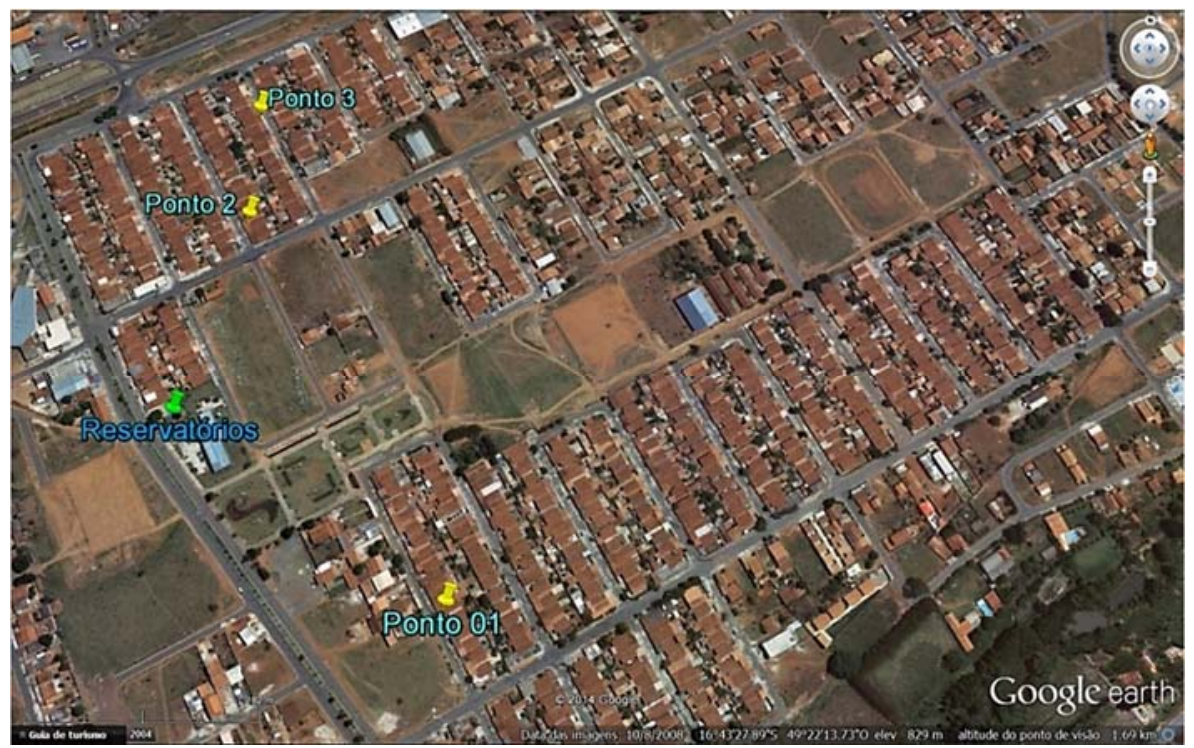

FIGURA 3: Pontos de instalação dos medidores de pressão do Conjunto Parque Santa Rita, Goiânia-GO. FONTE: Google Earth (2008).
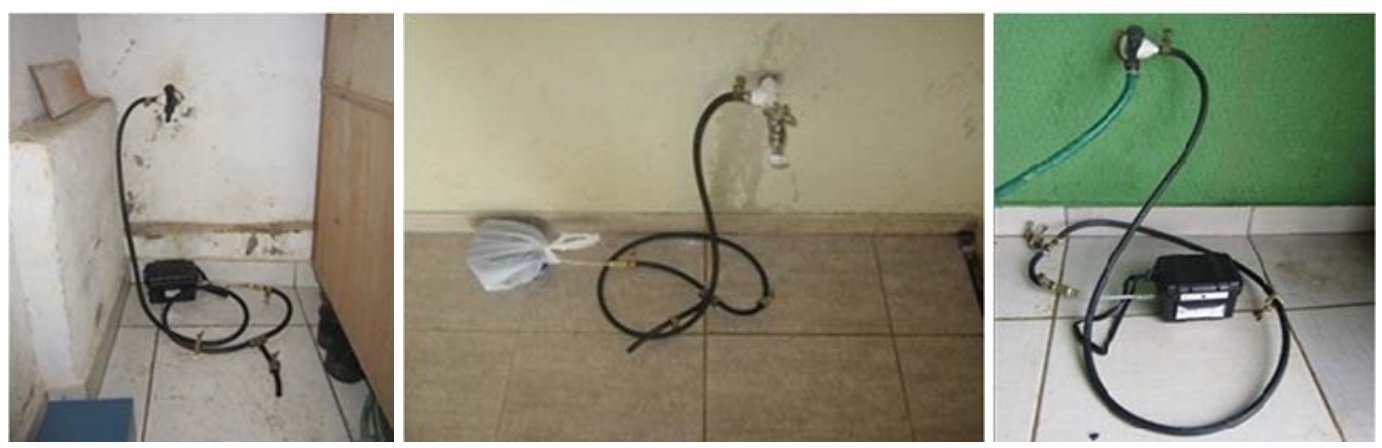

FIGURA 4: Medidores de pressão instalados nos pontos 01 (à esquerda), 02 (no meio) e 03 (à direita). 


\subsection{Calibração do modelo}

O modelo inverso aplicado visa a minimização dos desvios entre os valores de vazão e pressão observados durante o monitoramento e os valores simulados no EPANET. A função objetivo utilizada para a resolução do problema de calibração baseia-se no quadrado da diferença entre valores observados e simulados, conforme Equação 1.

O EPANET foi utilizado como um módulo auxiliar na avaliação hidráulica da rede, e o simulador hidráulico proposto por Soares (2003) (Figura 5) foi utilizado para o cálculo de pressão e vazão, considerando os vazamentos e demandas dirigidas pela pressão.

$$
\min _{Z} F O=\sum_{t=1}^{n^{P D}}\left[\sum_{j=1}^{n_{t}^{p}}\left(P_{t, j}-P_{t, j}^{*}\right)^{2} /\left(\sum_{i=1}^{n_{t}^{p}} P_{t, i}^{*} / n_{t}^{p}\right)^{2}+\sum_{j=1}^{n_{t}^{Q}}\left(Q_{t, j}-Q_{t, j}^{*}\right)^{2} /\left(\sum_{i=1}^{n_{t}^{Q}} Q_{t, i}^{*} / n_{t}^{Q}\right)^{2}\right]
$$

Em que:

$n^{P D}=$ número de padrões de demanda observados (-);

$n^{P}=$ número de padrões de demanda em que há observações de pressões $(-)$;

$n^{Q}=$ número de padrões de demanda em que há observações de vazões $(-)$;

$\boldsymbol{P}=$ cargas de pressão simuladas (mca);

$\boldsymbol{P}^{*}=$ cargas de pressão observadas (mca);

$\boldsymbol{Q}=$ vazões simuladas $(\mathrm{L} / \mathrm{s})$;

$Q^{*}=$ vazões observadas $(\mathrm{L} / \mathrm{s})$;

$\boldsymbol{Z}=$ vetor solução $(-)$.

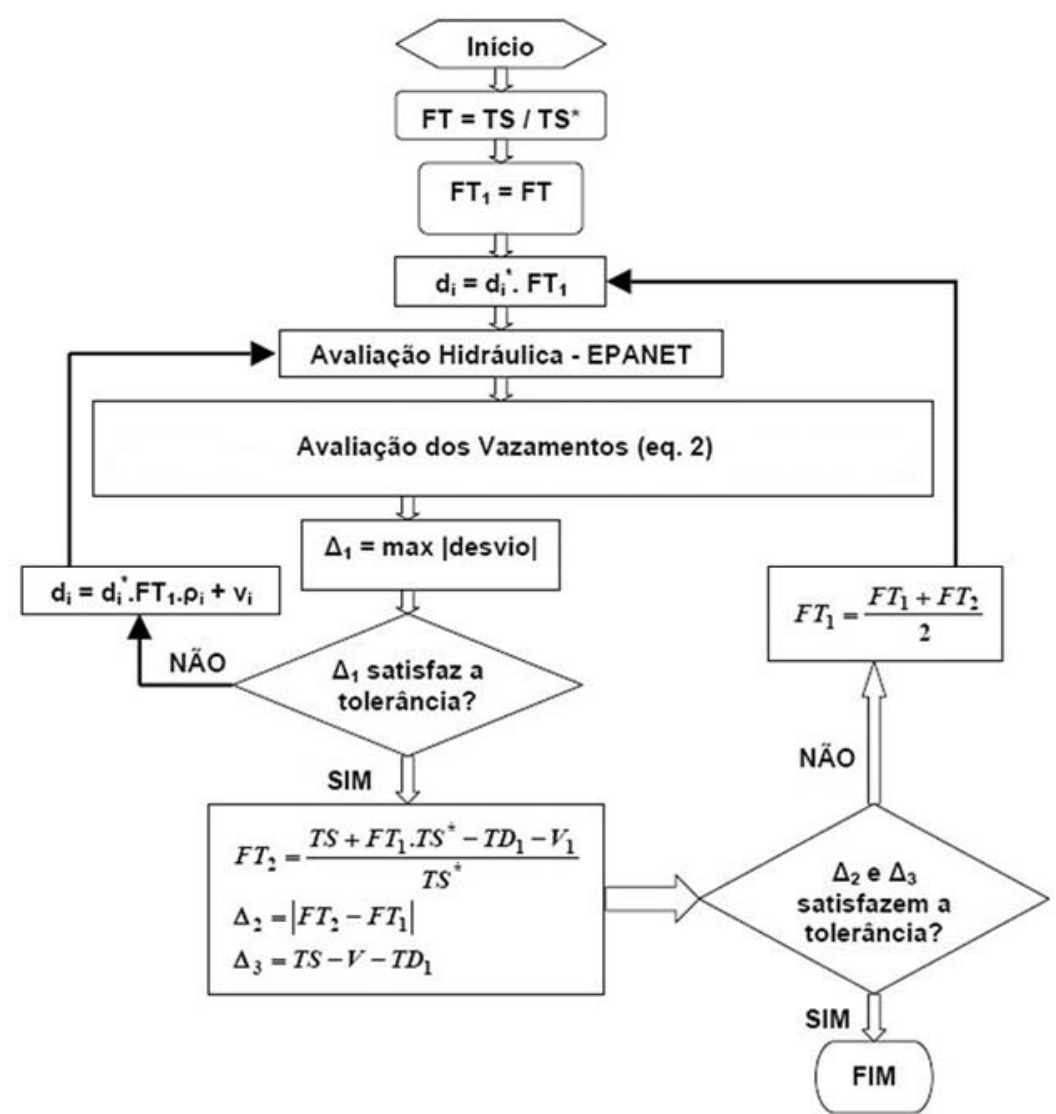

FIGURA 5: Fluxograma do processo iterativo proposto para avaliação hidráulica e determinação do fator de consumo para cada padrão (cenário) de demanda.

FONTE: Soares (2003). 
Na Figura 5, FT é um fator multiplicador de demanda (temporal), TS a vazão total abastecida, em L/s, $d_{i}$ a demanda no nó $i, T S^{*}$ a demanda total de referência, $\rho_{i} \quad 0$ fator multiplicativo da demanda total no nó $i, v_{i}$ o volume de vazamento no nó $i, V$ o volume total de vazamento na rede; TD a demanda total efetivamente abastecida, $\Delta_{1}$ o máximo desvio entre as pressões anteriores e presentes calculadas para cada nó, em mca, $\Delta_{2}$ a diferença entre os fatores multiplicativos atual e passado, e $\Delta_{3}$ a diferença da vazão total abastecida pelo volume total de vazamento e pela demanda total efetivamente abastecida. A tolerância estabelecida para os parâmetros $\Delta_{1}, \Delta_{2}$ e $\Delta_{3}$, foram $0,01 \mathrm{mca}, 0,01$ e $0,01 \mathrm{~L} / \mathrm{s}$, respectivamente.

Para o cálculo das perdas de água por vazamentos, foi adotada a Equação 2:

$$
V=\sum_{i=1}^{N} v_{i} \rightarrow v_{i}=\left(H_{i}-z_{i}\right)^{\beta} \sum_{j=1}^{M_{i}} \frac{\pi}{2} D_{i j} \theta_{i j} L_{i j} \quad \text { Eq. [2] }
$$

\section{Em que:}

$\boldsymbol{V}_{\boldsymbol{i}}=$ perda por vazamento nas tubulações ligadas ao nó $i\left(\mathrm{~m}^{3} / \mathrm{s}\right)$;

$\boldsymbol{H}_{\boldsymbol{i}}=$ carga piezométrica no nó $i$ (mca);

$\mathbf{z}_{i}=$ cota topográfica do nó $i(\mathrm{~m})$;

$\boldsymbol{\beta}=$ expoente de perda por vazamento (-);

$\boldsymbol{M}_{\boldsymbol{i}}=$ número total de tubulações ligadas ao nó $i(-)$;

$D_{i j}=$ diâmetro da tubulação entre os nós $i$ e $j(\mathrm{~m})$;

$\boldsymbol{L}_{i j}=$ comprimento da tubulação entre os nós $i$ e $j(\mathrm{~m})$;

$\theta_{i j}=$ taxa de perda por vazamento por unidade de superfície da tubulação entre os nós $i$ e $j\left(\mathrm{~m}^{1-\beta} / \mathrm{s}\right)$.

As variáveis de decisão que compuseram o vetor solução $(Z)$ foram: a rugosidade $\left(\varepsilon_{1}\right.$ e $\left.\varepsilon_{2}\right)$ e os coeficientes de vazamentos $\left(\theta_{1}\right.$ e $\left.\theta_{2}\right)$ das zonas 1 e 2 , e o expoente de vazamentos ( $\beta$ ). Sendo assim, $Z=\left\{\varepsilon_{1}, \varepsilon_{2}, \theta_{1}, \theta_{2}, \beta\right\}$. Cada vetor solução $(Z)$ foi utilizado no EPANET para o cálculo das demandas e pressões simuladas. Para a calibração foi utilizado o AG tipo Steady-State, devido seu menor tempo computacional. Neste tipo de AG somente os quatro piores vetores solução passam pelos operadores genéticos.

\section{RESULTADOS E DISCUSSÕES}

\subsection{Análise dos dados monitorados}

Para avaliar o padrão de consumo no setor, foi determinada a faixa de demanda de consumo durante o período de monitoramento referente ao perfil diário de consumo para o período de seis semanas (Figura 6). A partir dos dados coletados, foram traçadas as curvas das vazões mínima, média e máxima, e a fim de descartar pequenos ruídos, foi aplicado uma média móvel de dez minutos. As curvas obtidas refletiram um padrão de consumo bem definido e observouse que os valores de consumo mais baixos foram encontrados durante a madrugada, tendo seu início por volta das $6 \mathrm{h00}$ e alcançando maiores valores por volta das 10h00. Sendo assim, o padrão avaliado demonstrou o comportamento de um setor residencial, obtendo-se uma vazão média diária de 3,4 L/s (Figura 7).

Além disso, nota-se que existe um consumo de aproximadamente 0,9 L/s na madrugada, entre $02 \mathrm{h00}$ e 05h00, que pode ser explicado pelo abastecimento das caixas d'água residenciais e/ou pela ocorrência de vazamentos na rede. Entretanto, para verificar se realmente há consumo durante este período, seria necessário realizar o monitoramento da demanda de consumo por residência.

Foram construídas as curvas de variação da carga de pressão para cada ponto monitorado entre os dias 3 e 6 de maio de 2014, para fins de análise (Figuras 8 a 10). Pode-se observar em todos os pontos monitorados, que não houve grande variação de pressão ao longo do dia, sendo observados apenas breves picos de aumento ou redução de pressão. Em sistemas de porte maiores, o aumento do consumo resulta na redução da pressão, ocorrendo assim um comportamento inverso entre essas variáveis. Porém, o que pode ser observado no sistema em estudo, é que no período da manhã, quando inicia o consumo pelos residentes, ocorre um aumento da pressão ao invés de sua redução. Isso pode ser explicado pelo fato da rede ser relativamente pequena e, assim, o início do consumo causa uma perturbação na rede, resultando na variação da pressão em todos os pontos, caracterizando um regime transitório. 


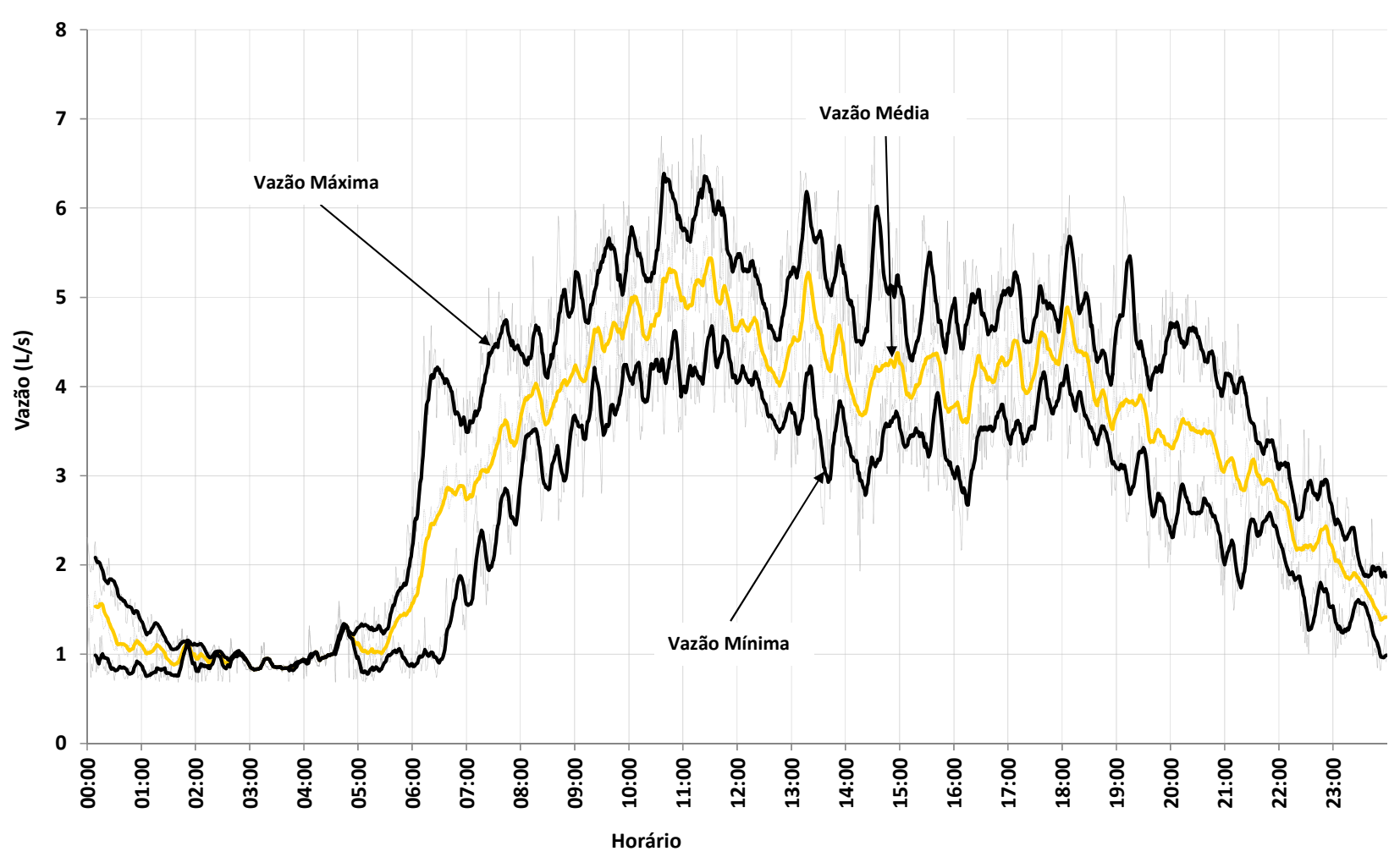

FIGURA 6: Faixa de demanda de consumo na zona alta da rede de abastecimento de água.

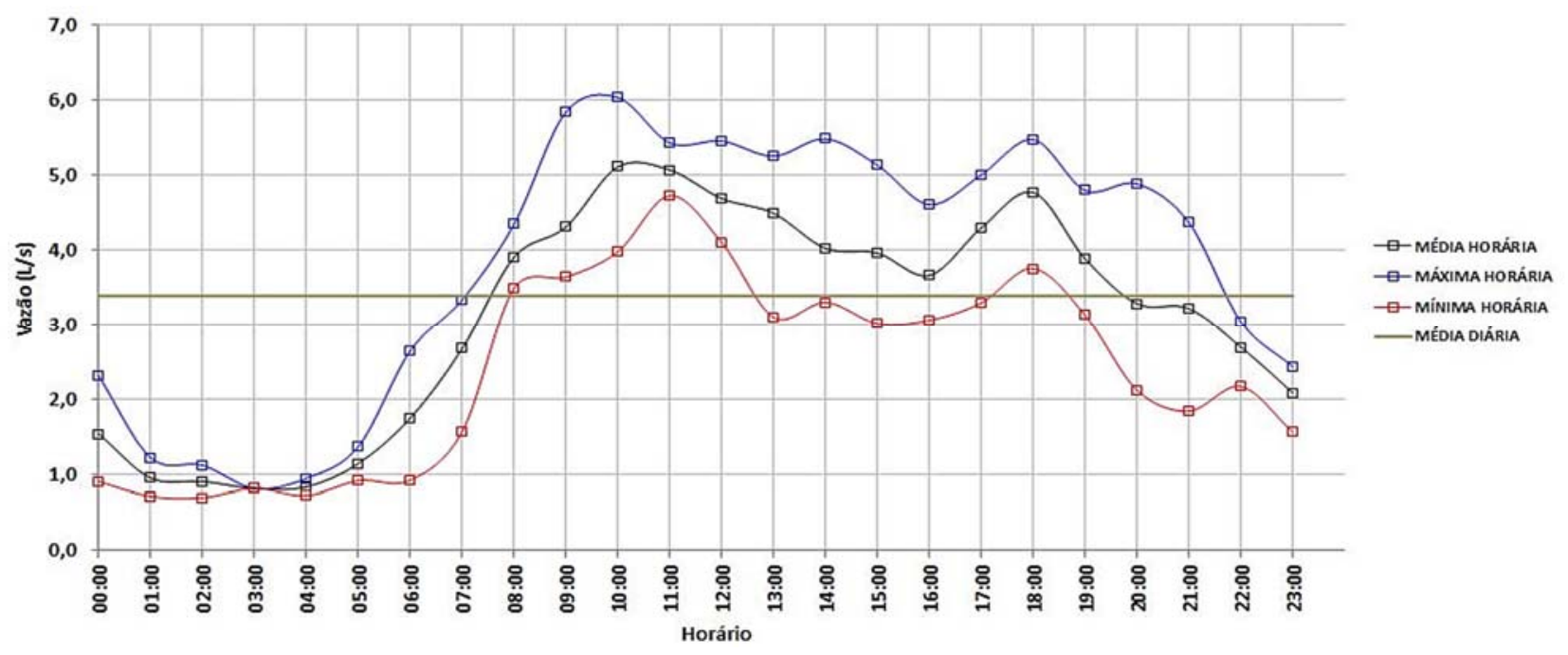

FIGURA 7: Curvas de vazões horárias mínima, média e máxima. 

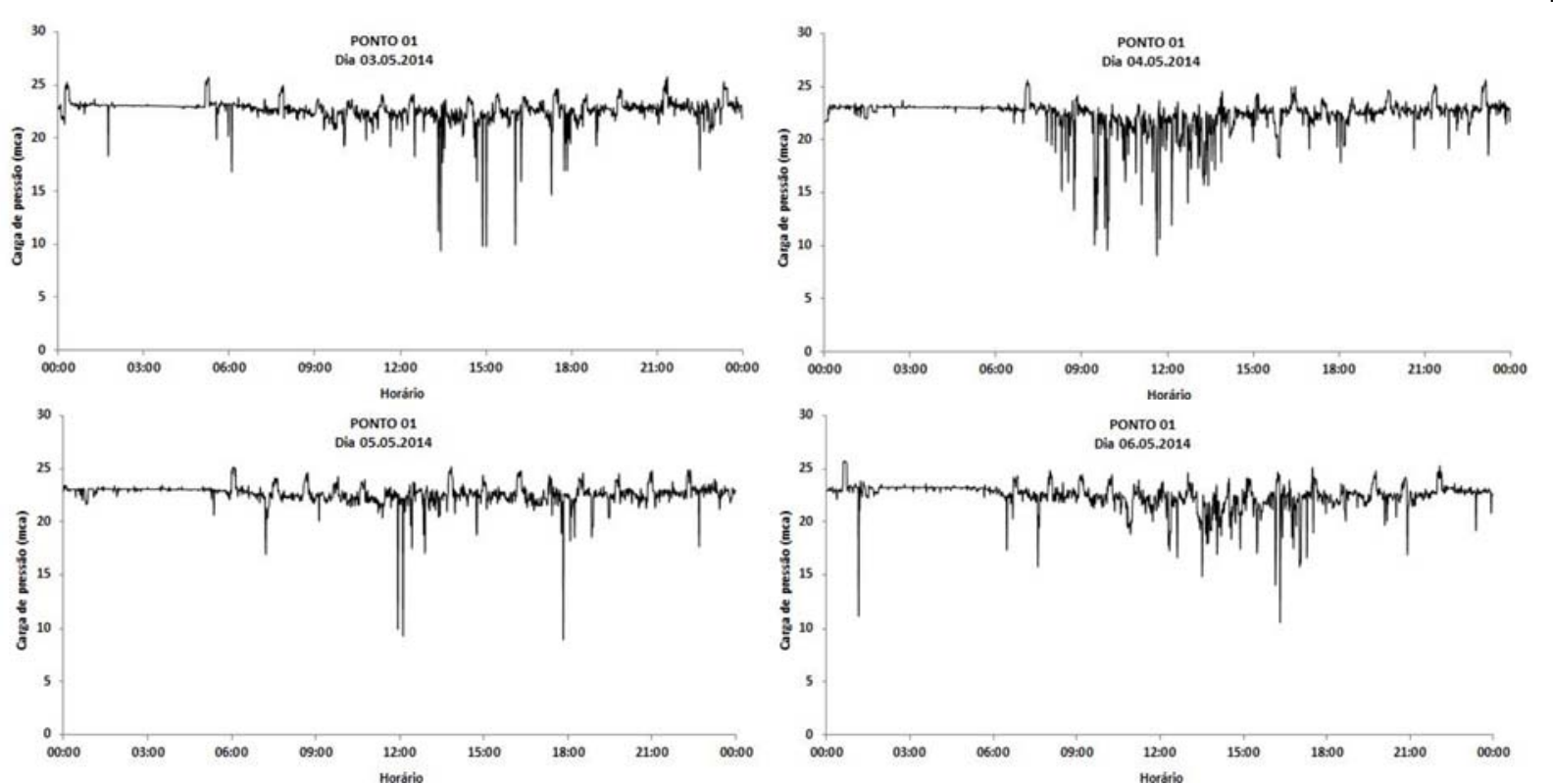

FIGURA 8: Curvas de cargas de pressão monitoradas no ponto 1 entre os dias 3 e 6 de maio de 2014.
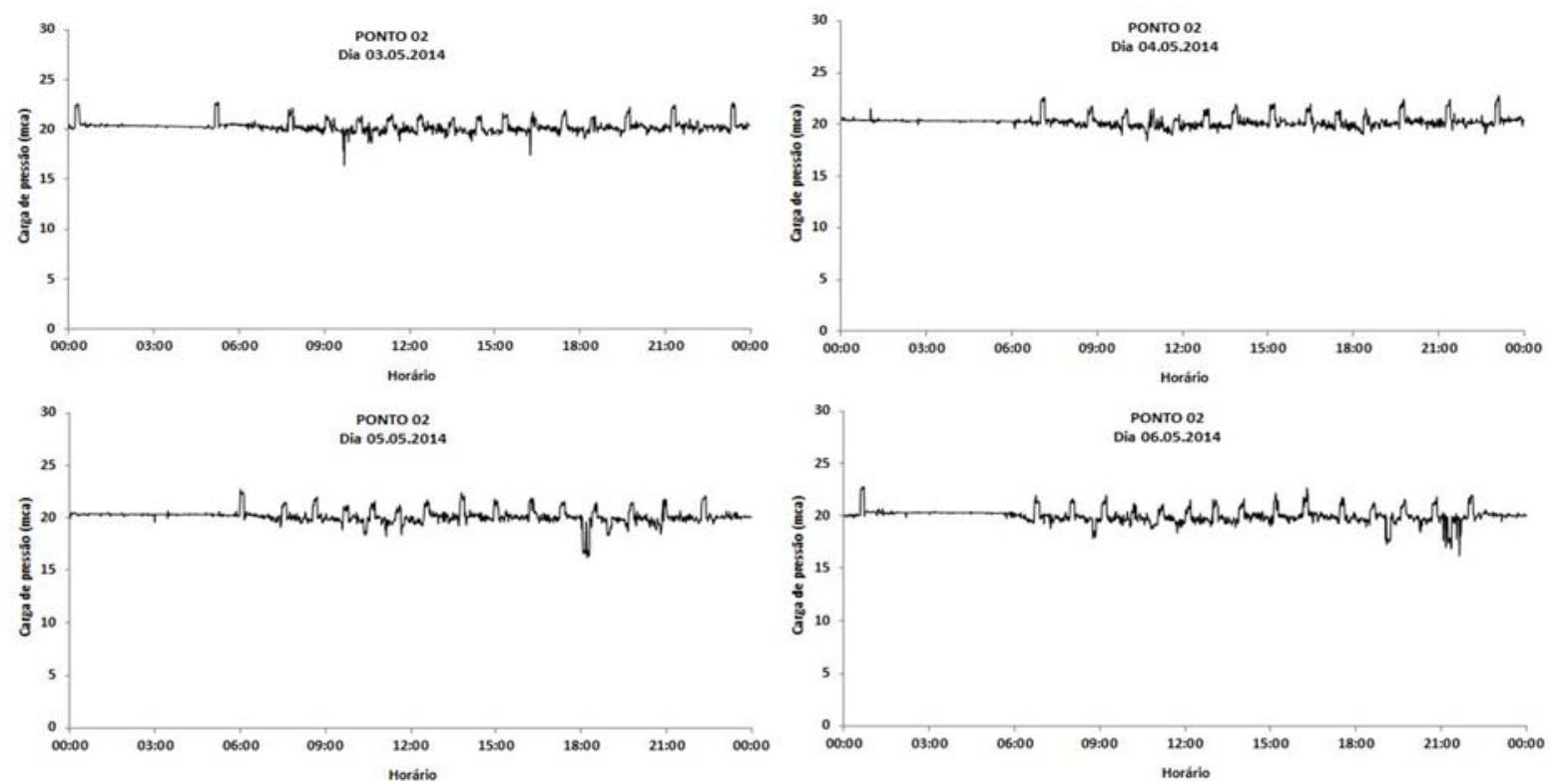

FIGURA 9: Curvas de cargas de pressão monitoradas no ponto 2 entre os dias 3 e 6 de maio de 2014.
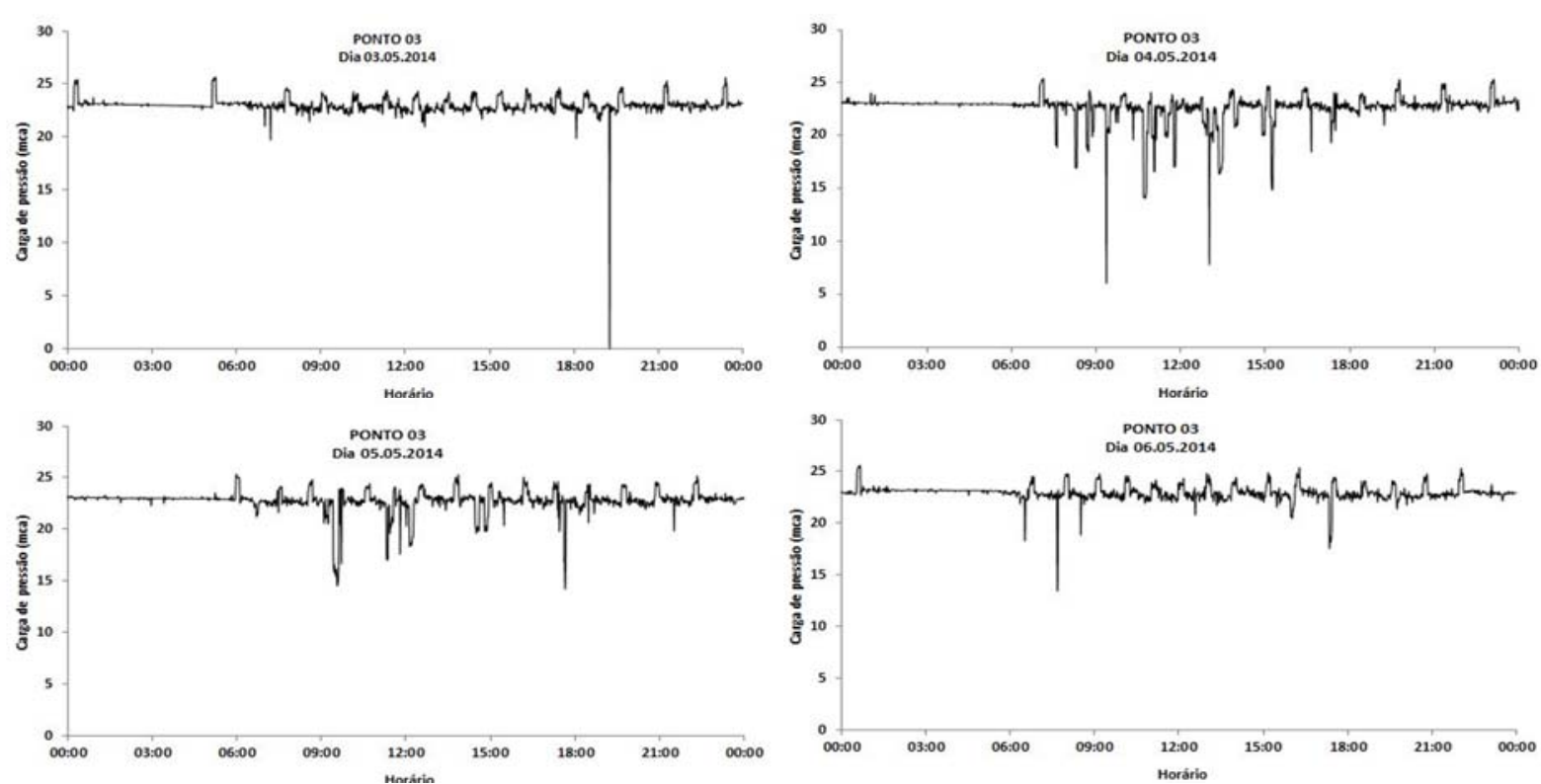

FIGURA 10: Curvas de cargas de pressão monitoradas no ponto 3 entre os dias 3 e 6 de maio de 2014. 


\subsection{Modelagem hidráulica}

Para a realização das simulações foi utilizado o modelo hidráulico da zona alta da rede de abastecimento do setor elaborado no software EPANET (Figura 11). A rede possui 35 nós e apresenta tubos de PVC com diâmetros que variam entre 50 e $150 \mathrm{~mm}$. Os nós monitorados utilizados para as análises de calibração foram aqueles localizados próximos dos pontos de monitoramento, sendo eles os nós 33, 10 e 8, para os pontos 1, 2 e 3, respectivamente. Para as simulações, foi necessário o cálculo do consumobase em cada nó da rede. Segundo Buchberger e Wu (1995), a demanda de consumo em residências não se dá de maneira contínua durante todo o dia, assumindo um padrão descontínuo, podendo ser representada por pulsos retangulares de vazão com intensidade e duração aleatórias. Entretanto, e como não foi possível monitorar a vazão de consumo em cada residência, foi considerado que o consumo-base de cada residência é a média da vazão distribuída no setor dividido para cada ligação. Na Tabela 3, são apresentados os valores de vazões mínima, média e máxima e seus respectivos consumo-base.

Devido ao fato de que o modelo EPANET é aplicável somente para escoamentos turbulentos, foi necessário fazer a verificação do número de Reynolds para cada trecho da rede e em distintos instantes do dia. Verificou-se que o único cenário em que ocorre o escoamento turbulento em todas as tubulações é o cenário de consumo máximo, podendo este ser aplicado ao modelo do EPANET. Portanto, o cenário utilizado nas análises de calibração foi o de maior vazão média $(5,11 \mathrm{~L} / \mathrm{s})$, observada às $10 \mathrm{~h}$ da manhã.

Além disso, deve-se destacar que a fórmula indicada para o cálculo do fator de atrito para escoamento turbulento liso, como ocorre nos tubos de PVC sem incrustação, é a de Blasius, não codificada no EPANET e que depende somente do número de Reynolds.

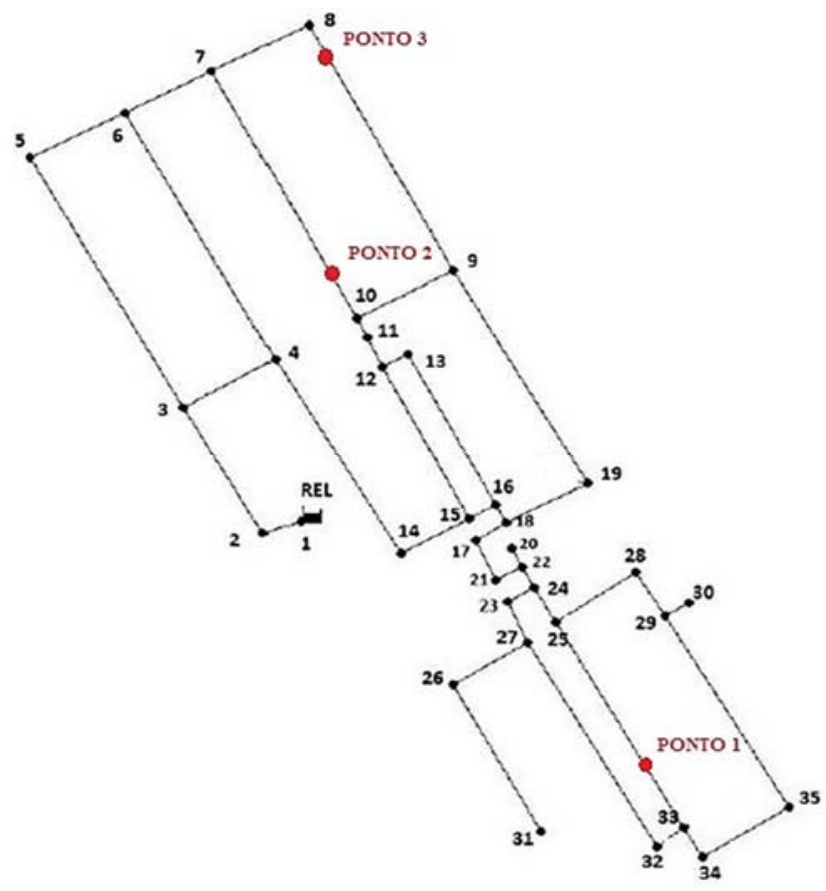

FIGURA 11: Modelo da rede de abastecimento de água da Zona Alta do Conjunto Parque Santa Rita.

TABELA 3: Consumo-base nos cenários de vazão mínima, média e máxima.

\begin{tabular}{ccc} 
Cenário & Vazão fornecida $\left(\mathbf{m}^{\mathbf{3}} \mathbf{\text { dia }}\right)$ & Consumo-base $\left(\mathbf{m}^{\mathbf{3}} /\right.$ ligação.dia) \\
\hline Vazão mínima & 232,96 & 0,6507 \\
\hline Vazão média & 302,97 & 0,8463 \\
\hline Vazão máxima & 369,14 & 1,0311 \\
\hline
\end{tabular}


3.3. Calibração do modelo hidráulico via algoritmos genéticos

Para a calibração do modelo, foi adotado um intervalo para as variáveis de decisão do vetor solução (Z). Dois setores foram estabelecidos na Zona Alta do Parque Santa Rita, sendo o primeiro (setor 1) com tubulações de 100 e 150 mm, e o segundo (setor 2) com tubulações de $50 \mathrm{~mm}$. Para a rugosidade absoluta, $\varepsilon$, foi adotado um intervalo de acordo com o material das tubulações (PVC: $0,01<\varepsilon<0,5 \mathrm{~mm}$ ); a faixa de valores do coeficiente de perda, $\theta$, foi a mesma para os dois setores $(0,0001$ a 1,0). 0 coeficiente de perda, $\beta$, foi considerado homogêneo para toda a Zona Alta, por se tratar de rede com mesmo material para as tubulações; sua variação nas análises de calibração foi de 0,50 a 1,20 .

Para a resolução do problema de calibração via algoritmos genéticos, são utilizadas sementes aleatórias que geram uma população de " $\mathrm{n}$ " indivíduos, sendo cada indivíduo um vetor solução (Z). Na calibração realizada neste trabalho, foram geradas cinco populações com 50 indivíduos cada, sendo o critério de parada a realização de 100 gerações. A Figura 12 mostra a variação da função objetivo para cada população do AG. A partir de 30 gerações os valores da função objetivo se tornam constante. A população 3 foi a que obteve o menor valor da função objetivo $(F O=0,016098)$. $O$ vetor $Z$ que resultou no menor valor da função objetivo obteve os seguintes valores para as variáveis de decisão: $\varepsilon_{1}=0,46 \mathrm{~mm}$; $\varepsilon_{2}=0,01 \mathrm{~mm} ; \theta_{1}=2,9 \times 10 \mathrm{E}-8 ; \theta_{2}=2,5 \times 10 \mathrm{E}-8$; $\beta=0,55$.
Com as variáveis de decisão encontradas (vetor solução Z), foi executada a simulação hidráulica no modelo de Soares (2003) para a comparação entre as cargas de pressão observadas e simuladas. A Tabela 4 mostra os valores das cargas de pressão simuladas e observadas para os três pontos de monitoramento. $\mathrm{O}$ erro absoluto encontrado é menor do que 1,5 m.c.a., o que é considerado aceitável para uma boa calibração do modelo indicando boa consistência do modelo. Foram calculadas as perdas de água por vazamento na rede a partir do modelo hidráulico de Soares (2003), que parte do princípio de que as perdas reais devem ser calculadas pela Equação [2] e subtraídas da vazão total fornecida, conforme o fluxograma da Figura 5. Os valores calculados de vazão fornecida e perdas por vazamento na Zona Alta são mostrados na Tabela 5 e na Figura 13. 0 erro relativo entre a vazão fornecida observada (266,510 $\left.\mathrm{m}^{3} / \mathrm{dia}\right)$ e a vazão fornecida simulada (267,144 $\left.\mathrm{m}^{3} / \mathrm{dia}\right)$ - soma entre consumo simulado e perda por vazamento - resultou em $0,24 \%$.

$\mathrm{O}$ volume de perdas reais representa menos de $1 \%$ do volume total distribuído, sendo um valor baixo para um setor de rede de abastecimento de água. Isso se deve ao fato da rede ser relativamente nova e operar com folga, registrando valores de vazões e pressões baixas. As perdas encontradas podem ocorrer devido a pequenos vazamentos distribuídos na rede ou nos ramais de cada residência. Além disso, deve-se destacar que as análises reportadas neste trabalho não levam em consideração as perdas aparentes, que podem elevar o percentual final de perdas de água obtido pela companhia de saneamento.

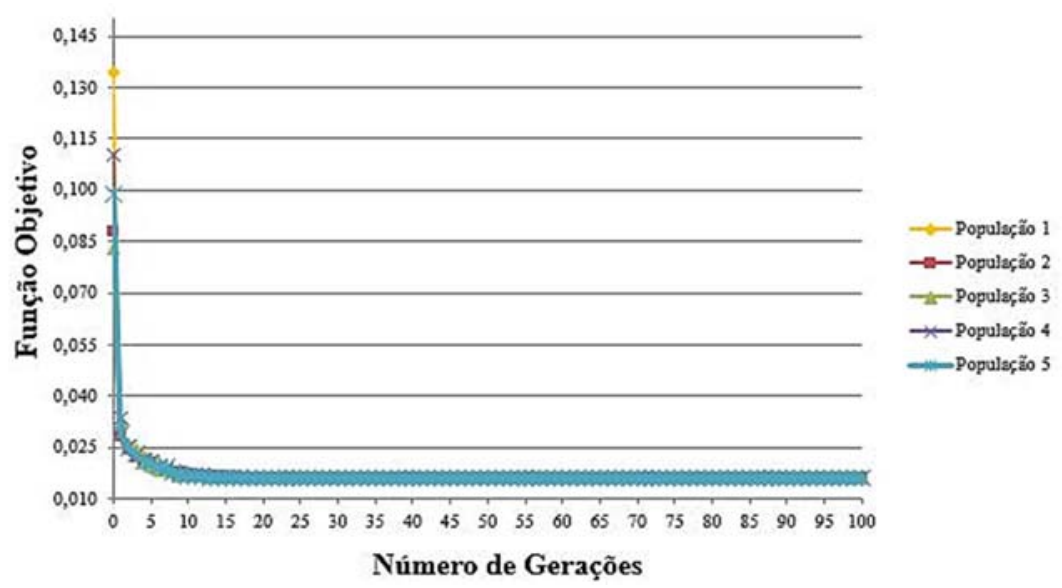

FIGURA 12: Variação da função objetivo FO para cada população. 
TABELA 4: Cargas de pressão simuladas e observadas.

\begin{tabular}{|c|c|c|c|c|c|c|c|c|c|}
\hline \multirow[t]{2}{*}{ População } & \multicolumn{3}{|c|}{$\begin{array}{l}\text { Carga de pressão } \\
\text { observada (mca) }\end{array}$} & \multicolumn{3}{|c|}{$\begin{array}{c}\text { Carga de pressão } \\
\text { simulada (mca) }\end{array}$} & \multicolumn{3}{|c|}{ Erro absoluto (mca) } \\
\hline & Nó 8 & Nó 10 & Nó 33 & Nó 8 & Nó 10 & Nó 33 & Nó 8 & Nó 10 & Nó 33 \\
\hline 1 & \multirow{5}{*}{20,13} & \multirow{5}{*}{22,70} & \multirow{5}{*}{22,13} & 20,06 & 21,22 & 22,28 & 0,07 & 1,48 & 0,15 \\
\hline 2 & & & & 20,12 & 21,21 & 22,31 & 0,01 & 1,49 & 0,18 \\
\hline 3 & & & & 20,10 & 21,21 & 22,27 & 0,03 & 1,49 & 0,14 \\
\hline 4 & & & & 20,09 & 21,21 & 22,30 & 0,04 & 1,49 & 0,17 \\
\hline 5 & & & & 20,13 & 21,22 & 22,32 & 0,00 & 1,48 & 0,19 \\
\hline
\end{tabular}

TABELA 5: Vazões observadas e simuladas

\begin{tabular}{|c|c|c|c|}
\hline Horário & $\begin{array}{l}\text { Vazão fornecida } \\
\text { observada (L/s) }\end{array}$ & $\begin{array}{c}\text { Consumo simulado } \\
\text { (L/s) }\end{array}$ & $\begin{array}{c}\text { Perda por vazamento } \\
(\mathrm{L} / \mathrm{s})\end{array}$ \\
\hline $00: 00$ & 1,537 & 1,5146 & 0,0298721 \\
\hline 01:00 & 0,953 & 0,930543 & 0,0299437 \\
\hline $02: 00$ & 0,909 & 0,88654 & 0,0299478 \\
\hline 03:00 & 0,816 & 0,793534 & 0,0299559 \\
\hline 04:00 & 0,837 & 0,814535 & 0,0299541 \\
\hline 05:00 & 1,146 & 1,12356 & 0,0299235 \\
\hline $06: 00$ & 1,749 & 1,72662 & 0,0298387 \\
\hline 07:00 & 2,677 & 2,65476 & 0,0296553 \\
\hline 08:00 & 3,902 & 3,88002 & 0,0293108 \\
\hline 09:00 & 4,307 & 4,28512 & 0,0291708 \\
\hline $10: 00$ & 5,107 & 5,08536 & 0,0288555 \\
\hline $11: 00$ & 5,063 & 5,04135 & 0,0288742 \\
\hline $12: 00$ & 4,685 & 4,66323 & 0,0290283 \\
\hline $13: 00$ & 4,487 & 4,46518 & 0,0291044 \\
\hline $14: 00$ & 4,020 & 3,99805 & 0,0292714 \\
\hline $15: 00$ & 3,957 & 3,93503 & 0,0292926 \\
\hline $16: 00$ & 3,660 & 3,63796 & 0,0293883 \\
\hline $17: 00$ & 4,293 & 4,27112 & 0,0291759 \\
\hline $18: 00$ & 4,764 & 4,74226 & 0,0289971 \\
\hline 19:00 & 3,882 & 3,86001 & 0,0293174 \\
\hline $20: 00$ & 3,282 & 3,25988 & 0,0294999 \\
\hline 21:00 & 3,216 & 3,19386 & 0,0295183 \\
\hline $22: 00$ & 2,696 & 2,67376 & 0,0296509 \\
\hline $23: 00$ & 2,085 & 2,06267 & 0,0297797 \\
\hline Total $\left(\mathrm{m}^{3}\right)$ & 266,510 & 264,598 & 2,546 \\
\hline
\end{tabular}

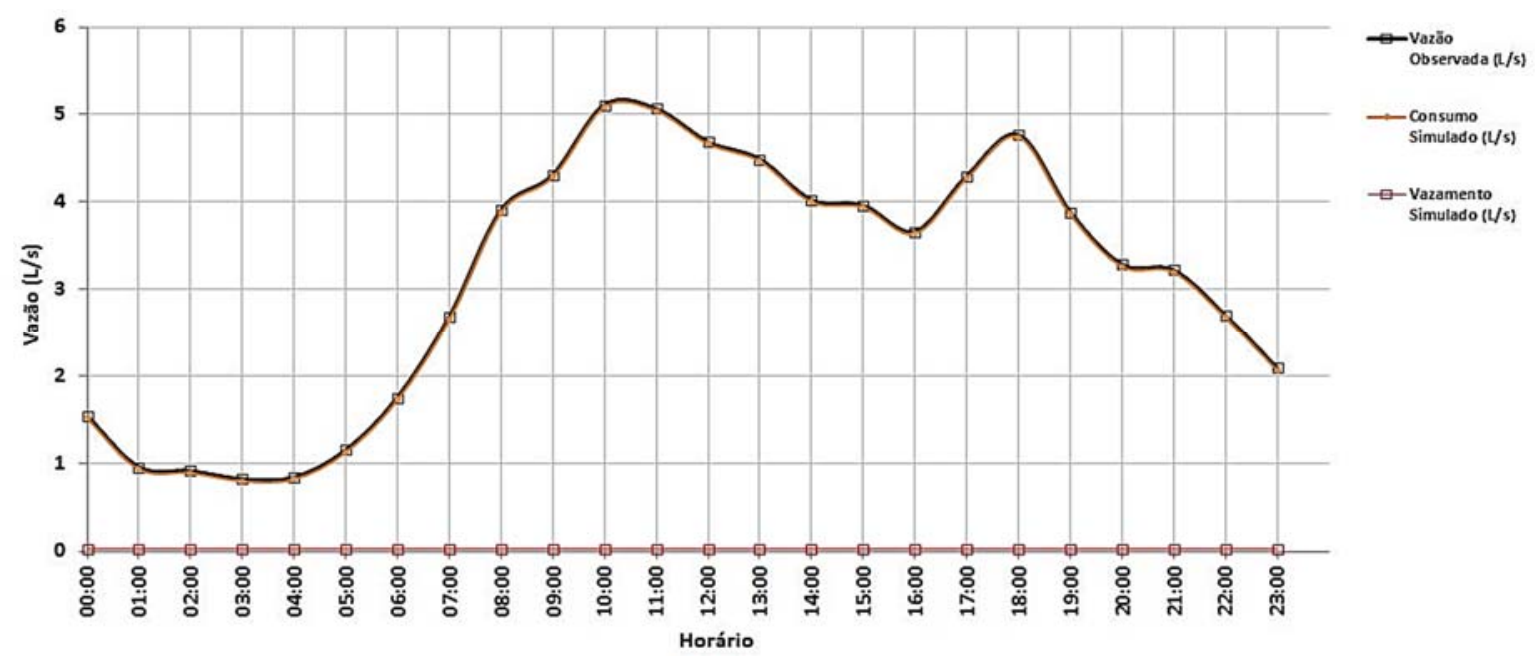

FIGURA 13: Curva de vazão fornecida observada, consumo e perdas reais simuladas para a Zona Alta do Setor Parque Santa Rita. 


\section{CONCLUSÕES E RECOMENDAÇÕES}

O trabalho apresentado consistiu da calibração do modelo hidráulico de uma rede de abastecimento real localizada em Goiânia, Goiás. Para tanto, foi utilizada a metodologia desenvolvida por Soares (2003), que utiliza o programa EPANET para a realização das simulações hidráulicas e resolve o problema de calibração por meio dos Algoritmos Genéticos.

O setor Parque Santa Rita apresenta um padrão de consumo bem definido e característico de um setor predominantemente residencial. Porém, e diferentemente do que se observa usualmente nos sistemas de grande porte, as curvas de pressão não possuem comportamento inverso ao da vazão e não possuem um padrão bem definido. Devido ao fato do consumo de água em residências ser representado por pulsos, recomenda-se que em trabalhos futuros que se realize a medição contínua do consumo residencial para cada ponto de monitoramento.

A calibração do modelo hidráulico apresentou resultados satisfatórios, uma vez que os desvios entre os dados simulados e observados em campo foram muito baixos, representando satisfatoriamente a rede estudada. Entretanto, deve-se considerar que o modelo hidráulico proposto não se adapta a sistemas com vazões baixas, nos quais as tubulações operam sob escoamento não turbulento por longos períodos do dia.

O sistema em estudo encontra-se superdimensionado, apresentando dados de vazão e pressão relativamente baixos, devido ao fato de se encontrar no início do horizonte de projeto para o qual foi projetado. $O$ volume de perdas reais determinado é consideravelmente baixo e, portanto, não há necessidade imediata da realização de campanhas de redução de perdas reais na área de estudo. Porém, é importante que haja continuidade do monitoramento no setor, e recomenda-se a análise do consumo que ocorre de madrugada para verificar se este consiste de vazamentos na rede/ramais ou de abastecimento dos reservatórios domiciliares.

\section{AGRADECIMENTOS}

À empresa SANEAGO, pela autorização e apoio nas investigações de campo, e disponibilização de informações e dados referentes à área de estudo; ao Conselho Nacional de Desenvolvimento Científico e Tecnológico (CNPq), pela bolsa de estudos concedida à primeira autora e pelo apoio financeiro aos projetos "Análise e Controle de Sistemas de Distribuição de Água via Modelagem Computacional" (Edital MCT/CNPq 14/2010 - Universal) e "Análise e Modelagem de Efeitos Dinâmicos e Cavitação durante Transitórios Hidráulicos em Sistemas de Distribuição de Água" (Chamada Universal MCTI/CNPq 14/2013). Os autores agradecem, também, aos revisores pelo esforço dedicado ao melhoramento do artigo.

\section{REFERÊNCIAS BIBLIOGRÁFICAS}

BUCHBERGER, S. G.; WU, L. Model for instantaneous residential water demands. Journal of Hydraulic Engineering. p 232 - 246, 1995.

EVANGELISTA, R. M. Implantação de programa de redução de perdas em sistemas de distribuição de água. 2004. Dissertação (Mestrado em Engenharia Civil) Faculdade de Engenharia Civil, Arquitetura e Urbanismo, Universidade Estadual Campinas - UNICAMP, São Paulo, 2004.

GARCIA, E. H.; LIMA, G. S.; GHISLENI, G.; DARONCO, G. C.; DALMAS, R. R. O. Sistema de abastecimento de água. Estudo de caso: Redentora - RS. In: II ENCONTRO NACIONAL DE ESTUDANTES DE ENGENHARIA CIVIL, 2013, Florianópolis, Santa Catarina.

GOLDBERG, D.E. Genetic Algorithms in Search, Optimization and Machine Learning. Addison-Wesley, Reading Massachusetts, 1989.

LACERDA, E. G.; CARVALHO, A. L. F. Introdução aos algoritmos genéticos. In: GALVÃO, C. O. (org); VALENÇA, M. J. S. (org): Sistemas Inteligentes: aplicações a recursos hídricos e sistemas ambientais. Ed. Universidade/UFRGS/ABRH, Porto Alegre, 1999.

NELDER, J.A.; MEAD, R. A simplex method for function minimization. Computer Journal, vol. 7, n. 4, p. 308-313, 1965.

ROSSMAN, L.A. EPANET 2 Users Manual, US Environmental Protection Agency, 2000. 
SANTOS, A. C. N. Estudo de calibração de uma rede de distribuição de água de Itajubá - MG utilizando os algoritmos genéticos. 2010. Dissertação (Mestrado em Ciências em Meio Ambiente e Recursos Hídricos) Universidade Federal de Itajubá - UNIFEI, Itajubá, 2010.

SILVA, F. G. B.; REIS, L. F. R. Calibração de redes de distribuição de água com algoritmos genéticos aplicada a uma rede hipotética. In: VI Simpósio Ítalo Brasileiro de Engenharia Sanitária e Ambiental, 2002, Vitória, Espírito Santo.

SILVA, F. G. B.; REIS, L. F. R.; SOARES, A. K.; FORMIGA, K. T.; CARRIJO, I. B. Modelo de calibração de rede de distribuição de água considerando fugas: estudo de caso do setor real Monte Carlo. In: XV Simpósio Brasileiro de Recursos Hídricos, 2003, Curitiba, Paraná.

SOARES, A. K. Calibração de modelos de rede de distribuição de água para abastecimento considerando vazamentos e demandas dirigidas pela pressão. $178 \mathrm{f}$. Dissertação (Mestrado em Hidráulica e Saneamento) Escola de Engenharia de São Carlos, Universidade de São Paulo - USP, São Carlos, 2003.

SOARES, A. K.; REIS, L. F. R. Calibração de modelos de redes de distribuição de água utilizando modelo de simulação hidráulica dirigido pela pressão (MSHDP) e Método Híbrido AG-Simplex. Revista Brasileira de Recursos Hídricos, vol. 9, n. 2, p. 85-96, 2004.

SOARES, A. K.; CHEUNG, P. B.; REIS, L. F. R.; SANDIM, M. R. Avaliação das perdas físicas de um setor da rede de abastecimento de água de Campo Grande - MS via modelo inverso. Revista Engenharia Sanitária e Ambiental, vol. 9, n. 4, 2004.

TSUTIYA, M. T. Abastecimento de água. 3. Ed. São Paulo: 2006. $643 \mathrm{p}$.

VIEGAS, J. V.; PAZ, M. F.; GASTALDINI, M. C. C.; DANIELLE, R. D.; BARROSO, L. B. Simulação do comportamento hidráulico de redes de abastecimento de água utilizando o modelo EPANET. In: XXVIII Congresso Interamericano de Ingenierña Sanitaria y Ambiental. Cancún, México, 27 a 31 de outubro, 2002. 